Revista de la Escuela de Ciencias de la Educación, año 13, nRo. 12, vol. 2, julio a diciembre de 2017. Páginas 189192. ISSN 1851-6297. ISSN 2362-3349 (EN LíNEA). CIENCIA ENTRE TODXS: UNA PRODUCCIÓN COLECTIVA DESDE Y PARA LA EDUCACIÓN CIENTIFICA. LUCIA M. CONDENANZA.

\title{
CIENCIA ENTRE TODXS: UNA PRODUCCIÓN COLECTIVA DESDE Y PARA LA EDUCACIÓN CIENTÍFICA
}

\author{
Lucía M. Condenanza* \\ Universidad Nacional de La Plata, Argentina. \\ luciacondenanza@yahoo.com.ar
}

Recibido: 30/09/2016 Aceptado: 23/10/2016

\section{Resumen}

Se reseña el libro "Ciencia entre todxs. Tecnociencia en contexto social. Una propuesta de enseñanza", compilado por Alicia Massarini y Adriana Schnek, CABA: Paidós, 2015. La obra presenta un marco conceptual para el trabajo en la educación científica en distintos niveles educativos, que acompaña con propuestas de trabajo áulico para seis (6) problemáticas socio-científicas seleccionadas.

Palabras Clave: Educación científica -Tecnociencia - Propuestas de enseñanza - Didáctica.

\begin{abstract}
We present a review of the book titled "Ciencia entre todxs. Tecnociencia en contexto social. Una propuesta de enseñanza", edited by Alicia Massarini and Adriana Schnek, Paidós, CABA, 2015. The work shows a specific approach to science education in different educational levels, complemented by teachingproposals for six (6) selected issues related to science and society.
\end{abstract}

Profesora y Licenciada en Ciencias de la Educación (UNLP), Especialista en Currículum y Prácticas escolares en contexto (FLACSO), doctoranda en Ciencias de la Educación (UNLP). Becaria de la UNLP con lugar de trabajo en el Instituto de Investigaciones en Humanidades y Ciencias Sociales (IDIHCS, FaHCE-UNLP). Docente de Didáctica en la Facultad de Humanidades y Ciencias de la Educación (UNLP) y en el Instituto Superior de Formación Docente y Técnica $n^{\circ} 49$ (Brandsen, Bs. As.). Integrante del Grupo de Didáctica de las Ciencias (IFLYSIB, UNLP-CONICET). 
Revista de la Escuela de Ciencias de la Educación, año 13, nRo. 12, vol. 2, julio a diciembre de 2017. PÁginas 189192. ISSN 1851-6297. ISSN 2362-3349 (EN LINEA). CIENCIA ENTRE TODXS: UNA PRODUCCIÓN COLECTIVA DESDE Y PARA LA EDUCACIÓN CIENTIFICA. LUCIA M. CONDENANZA.

Key words: Science education - techno-science - teaching proposals.

"Una de las tareas del educador o la educadora progresista, a través del análisis político serio y correcto, es descubrir las posibilidades -cualesquiera que sean los obstáculos- para la esperanza (...)" (Freire, 1992, p.25)

Escribo esta reseña como lectora interesada en compartir "las posibilidades para la esperanza" que decía Freire; en este caso, vinculadas a una transmisión humanizada y democrática de la ciencia en la educación pública. Humanizada y democrática, en el sentido de anclarla en los intereses, motivaciones y consecuencias reales que implica el desarrollo científico. Democrática y politizada, en el sentido de reconocer la relación entre conocimiento y poder, y entre conocimiento, capital y poder.

Pocas veces encontramos la $x$, en tanto insignia de inclusión sexo-genérica a través del lenguaje, en trabajos científicos, y menos aún en producciones de científicos/as sobre la ciencia. Tal vez sea esto lo que primero llama la atención en el libro, y junto con la expresión "entre todxs", se abren interesantes preguntas para pensar la enseñanza y la democratización de la ciencia. Como podrán anticipar los y las lectoras, la democratización -no la divulgación- del conocimiento y de las decisiones y la soberanía recorren la obra como preocupación política, sociocientífica y pedagógica, acompañada de propuestas para el trabajo áulico.

El libro en cuestión surge de un trabajo colectivo desarrollado por el grupo "Ciencia entre todxs", el cual nació en 2009 y se dedica a la investigación-acción, la enseñanza y comunicación de distintas temáticas científicas y tecnológicas, desde una perspectiva de la complejidad. La autoría del libro corresponde a Alicia Massarini, Adriana Schnek, Beatriz Libertini, Érica Carrizo, Gonzalo Corti Bielsa, Guillermo Folguera, Nicolás José Lavagnino, Paula Lipko, trabajadores/as de diversas instituciones científicas y educativas del país. La variedad de espacios de inserción del grupo puede apreciarse a través de su página web: https://cienciaentretodos.wordpress.com/. La producción está organizada en dos partes: la primera, titulada "Cimientos y andamiajes para la enseñanza de las ciencias en el contexto latinoamericano", con cuatro capítulos referidos a las relaciones ciencia-sociedad, al pensamiento latinoamericano en ciencia y tecnología, la dimensión ambiental y al enfoque situado y complejo de la enseñanza respectivamente. En la segunda parte se presentan conceptos y propuestas de abordaje para seis problemáticas socio-científicas deliberadamente seleccionadas: los organismos genéticamente modificados, la enfermedad de Chagas, las nuevas tecnologías reproductivas, la identificación de personas mediante estudios genéticos, la clonación y las sustancias químicas en la producción de alimentos. Por último, se incluye una 
Revista de la Escuela de Ciencias de la Educación, año 13, nRo. 12, vol. 2, julio a diciembre de 2017. Páginas 189192. ISSN 1851-6297. ISSN 2362-3349 (EN LíNEA). CIENCIA ENTRE TODXS: UNA PRODUCCIÓN COLECTIVA DESDE Y PARA LA EDUCACIÓN CIENTIFICA. LUCIA M. CONDENANZA.

reflexión sobre la lectura, escritura y el habla en ciencia y tecnología como herramientas significativas para el diseño de estrategias de enseñanza. Coherente con la propuesta pedagógica que se pregona, los/as autores y autoras nos interpelan como lectores y lectoras curiosos/as, y en cada apartado de cada capítulo sugieren y comentan alguna bibliografía para ampliar y/o complejizar lo presentado.

“¿Cómo deseamos vincularnos socialmente y con el entorno natural?", se preguntan, y como respuesta nos interponen la necesidad de construir definiciones propias desde una perspectiva emancipadora de la educación y el conocimiento. El enfoque ciencia, tecnología y sociedad (CTS) difundido en los últimos años desde el campo académico -sobre todo en Estados Unidos y Europa- para el trabajo en educación científica no satisface las inquietudes que pueden surgir en estas latitudes. Por eso apuestan a la construcción de un enfoque "que incorpore las relaciones que se establecen entre la ciencia, la tecnología, la sociedad y el ambiente en nuestro particular contexto regional".

Un primer concepto que desarrollan es el de tecnociencia, dando cuenta de la "estrecha relación bidireccional que hay entre ciencia y tecnología desde la segunda mitad del siglo XX". Otro concepto que se desarrolla es el de problema científico, en comparación con el de problemas sociales. A diferencia de las propuestas de enseñanza más difundidas, los/as autores/as nos invitan a "navegar las complejas aguas del pluralismo", tomando los problemas sociales como punto de partida para la enseñanza de las ciencias. Para esto nos ofrecen criterios y herramientas concretas para el trabajo en el aula, que involucran necesariamente otros discursos más allá del científico.

La premisa del arraigo regional-latinoamericano de este enfoque propone recuperar el pensamiento de quienes escribieron "interesados en la construcción de sociedades más igualitarias", y postularon "una ciencia y una tecnología vinculadas a los problemas sociales y productivos regionales", autónoma tanto a nivel metodológico como en la definición de los temas prioritarios respecto de las agendas de los países centrales. Así, los/as autores/as se posicionan frente a las "innegables contradicciones relacionadas con la expansión de proyectos neoextractivistas" en la realidad regional actual para poner en vigencia el planteo de -entre otros- Varsavsky, Herrera y Sábato, científicos argentinos de los '60 y '70. En esta clave latinoamericana se considera también la dimensión ambiental del enfoque, analizando las especificidades regionales y los actores de las problemáticas ambientales locales en el marco de una poderosa trama de intereses internaciones y prácticas "poco amigables" con el ambiente.

La politicidad, tanto de la educación como de la actividad científica, es otro eje central en este enfoque. Quizá los contenidos vinculados con la naturaleza de la ciencia y los objetivos de la educación científica orientados a la participación ciudadana ofrezcan una plataforma para la lectura, apropiación e 
implementación de este enfoque. Un estudio realizado entre 2008 y 2012 en la provincia de Buenos Aires, muestra que los y las docentes, interpelados individualmente, enfatizan el desarrollo de la creatividad y el espíritu crítico como finalidad de la enseñanza de las ciencias, mientras que "en reflexiones grupales [surgen] la formación para la ciudadanía, la ciencia como cultura, la ciencia útil para la vida cotidiana", entre otras (Cordero y Mengascini, 2014). En este sentido, los escenarios colectivos de trabajo también pueden favorecer la construcción del enfoque propuesto en Ciencias entre todxs en la medida en que puedan ponerse en juego las representaciones sociales de los actores educativos. Esta idea alude al trabajo colectivo entre docentes, pero también y principalmente a la compleja tarea de ejercitar nuestra escucha con nuestros estudiantes en el proceso de enseñanza. En palabras de los/as autores/as, este enfoque implica una pedagogía de la complejidad, situada, indisciplinada.

Para finalizar, quiero compartir la definición de este libro como propuesta esperanzadora, no sólo porque nos proporciona un interesante marco teórico para pensar las prácticas de enseñanza, sino también -como ya se dijo-, nos ofrece herramientas para trabajar los contenidos escolares de ciencias desde un enfoque pedagógico democratizador. Las problemáticas seleccionadas conllevan un abordaje interdisciplinar, con la posibilidad de recuperar saberes que dialoguen con el discurso científico y son de interés y preocupación para nuestros jóvenes. Y por su parte, la perspectiva de la complejidad, que podría parecer inabordable, es convidada con consignas de trabajo viables en nuestras aulas, con sugerencias de diversos materiales de acceso libre. En ambos casos, nos proponen -una vez más- criterios y materiales desde los que re-crear la propuesta en función de los intereses y los sueños de cada comunidad escolar.

\section{Referencias bibliográficas}

- Cordero, S. y Mengascini, A. (2014). ¿Para qué educar en ciencias naturales, ambiente y salud hoy en Argentina?. Archivos de Ciencias de la Educación, 7. Recuperado de http://www.archivosdeciencias.fahce.unlp.edu.ar/article/view/Archivos07a04.

- Massarini, A. y Schnek, A. (2015).Ciencia entre todxs: tecnociencia en contexto social. Una propuesta de enseñanza. ( $1^{\circ}$ ed.). Ciudad de Buenos Aires: Paidós. 